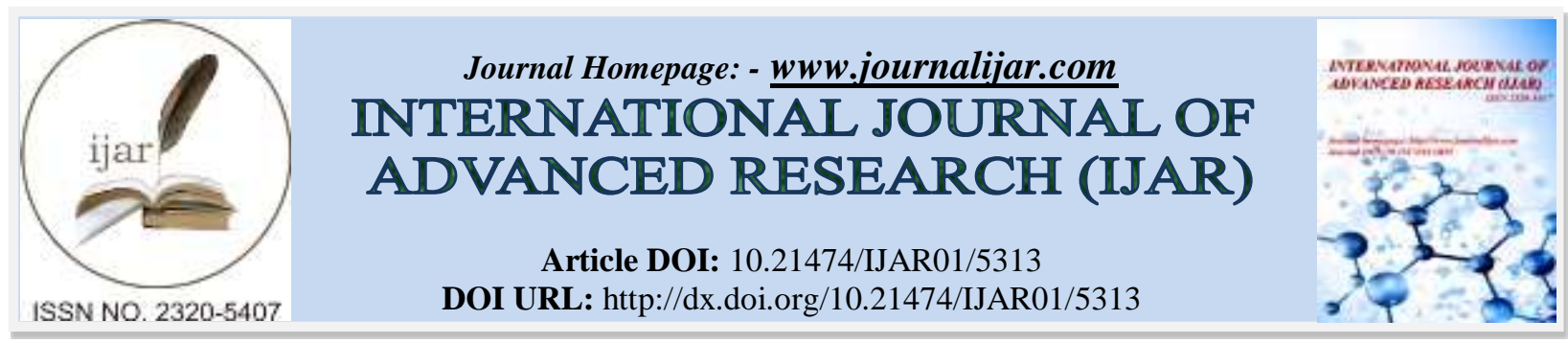

RESEARCH ARTICLE

\title{
LEVEL OF KNOWLEDGE ON CERVICAL CANCER, CERVICAL SCREENING AND ITS DETERMINANTS AMONG MINISTRY OF HEALTH PHC PHYSICIANS IN MAKKAH AL- MUKARRAMAH 2016, CROSS- SECTIONAL STUDY.
}

\author{
1. M.B.B.S, Family Medicine Resident. \\ Shaima'a Al Ahmadi ${ }^{1}$, Mulham Korani ${ }^{2}$ and Bakr Kalo ${ }^{3}$. \\ 2. Family Medicine Consultant. \\ 3. Family \& Community Medicine Consultant.
}

\section{Manuscript Info}

Manuscript History

Received: 03 July 2017

Final Accepted: 05 August 2017

Published: September 2017

Key words:-

Cervical cancer, cervical cancer screening, PHC physician's, knowledge.

\begin{abstract}
Objectives: To estimates the level of knowledge of cervical cancer, cervical screening and to identify their determinants among MOH PHC physicians in Makkah Al-Mokarramah city 2016.

Subjects and methods: A cross-sectional study was done among physicians working at Ministry of health primary health care centers, Makkah AL-Mokarramah A validated self-created self-administered questionnaire was used for data collection including (sociodemographic and physicians' knowledge about cervical cancer and its screening guidelines).

Results: The study included 137 physicians, with a mean age $39.7 \pm$ 8.8 years. Almost half of participants $(46.7 \%)$ were males, $43.1 \%$ were Saudi, $36.5 \%$ of PHCCs were JCI/CBAHI-accredited. Most participants $(89.1 \%)$ heard about Pap smear testing, while only $36.5 \%$ had an $\mathrm{MOH}$-protocol for cervical cancer screening, $33.6 \%$ ask asymptomatic female patients about screening for cancer cervix, $14.6 \%$ always refer female patients for screening for cancer cervix, $49.6 \%$ read about cervical screening guidelines last year, 26.3\% attended CME on cervical cancer and its screening last year. The most frequent correctly answered statements by PHC physicians about cancer cervix were related to cervical cancer is preventable $(78.8 \%)$ and history of sexually transmitted infections as a high-risk factor for cervical cancer (73.7\%). Overall, $31.4 \%$ of the physicians were knowledgeable regarding cancer cervix and its screening guidelines.

Conclusion: The overall knowledge of PHC physicians working at $\mathrm{MOH}$ inside Makkah AL-Mokarramah was suboptimal regarding cervical cancer and its screening guidelines.

PHC physicians should be encouraged toward regularly attending CME courses/workshops on prevention and management of cancer cervix.
\end{abstract}

Copy Right, IJAR, 2017,. All rights reserved.

\section{Introduction: -}

Cervical cancer is the fourth most common cancer in women, and the seventh overall, with an estimated 528,000 new cases and estimated 266,000 deaths worldwide in 2012, which account for $7.5 \%$ of overall cancer death. ${ }^{(1)}$ In 
Saudi Arabia, it's the eighth most common malignancy in Saudi females between 30 to 44 years of age and the fourth most common malignancy in non- Saudi nationals females, with incidence continue to be one of the lowest in the world. ${ }^{(2)}$

Human papillomavirus (HPV) is fundamental to the development of cervical neoplasia and can be detected in 99.7 percent of cervical cancer. ${ }^{(3)}$ There are 40 types identified for HPV with the subtypes 16 and 18 found mainly in cervical cancer. The epidemiology of HPV infection among females in Saudi Arabia is not fully comprehended due to the lack of database and limited publications regarding prevalence and genotype. ${ }^{(4-6)}$ Most of the HPV infections are transient and when it persists, the duration from initial infection to development of high-grade cervical neoplasia and invasive cancer take an average of 15 years. $^{(7)}$

Most of the risk factors for cervical cancer are associated with the increased risk of acquiring HPV infection. Early onset of sexual activity with two folds' increase in risk if the patient is less than eighteen years of age. Multiple sexual partners, which increases the risk up to three folds with six or more partners. ${ }^{(8)}$ Smoking ,in particular, increases the risk of squamous cell carcinoma of the cervix. ${ }^{(9)}$ Oral contraception use has been proclaimed to be associated with an increased risk of cervical cancer especially with increase duration of use. ${ }^{(8)}$ Other risk factors include; high-risk sexual partners, history of sexually transmitted infections, immunosuppression, low socioeconomic status, early age at first birth, increase parity and history of vulvar or vaginal squamous intraepithelial neoplasia or cancer. ${ }^{(10)}$

The two most common histological types are squamous cell carcinoma and adenocarcinoma with different HPV subtypes for each. ${ }^{(11)}$ Cervical cancer usually originates at transformation zone. ${ }^{(12)}$

Early cervical cancer is frequently asymptomatic, emphasizing the importance of screening. Irregular or heavy vaginal bleeding and post coital bleeding are the most common presentations. ${ }^{(13)}$

Physical examination should be performed in any female with suggestive symptoms, and any abnormal lesions should be biopsied. ${ }^{(14)}$ Diagnosis is made based on histological evaluation of a cervical biopsy, and cervical cytology should be performed to any women with suspected cervical cancer as it is the principle method for screening. ${ }^{(15)}$

After histological confirmation of diagnosis, a staging system must be implemented to assess disease staging. There are two aligned staging systems available, both of which use clinical rather than surgical criteria to determine disease stage; FIGO and TNM systems. ${ }^{(16,17)}$ For standard staging procedure, FIGO guidelines recommend several examinations for staging, but it is not mandatory to perform all of these tests on every patient e.g. physical examination, cervical cytology, an endoscopic procedure, etc. A Physical examination which includes pelvic examination through a speculum, bimanual, and rectovaginal examination for palpation and inspection of the primary tumor, uterus, vagina, and parametria, and examination for distant metastases by palpation of groin ,supraclavicular lymph nodes, and by examination of the right upper quadrant. ${ }^{(14)}$ Cervical biopsy either by colposcopy with directed cervical biopsy or cervical biopsy without colposcopy if a lesion is visible, endocervical curettage or conization. Endoscopic procedure using hysteroscope, cystoscope and proctoscope. Imaging studies used to evaluation for urinary tract obstruction using IVP, MRI or CT, and imaging with a plain chest radiograph and radiograph of the skeleton for metastases. ${ }^{(18)}$ Other laboratory and surgical procedure might be used to identify the presence of lymph node and distant metastasis.

Regarding management of cervical cancer, it can be done using different modalities of treatment such as chemotherapy, radiation and surgically depending on disease stage. ${ }^{(19)}$

Due to the impact of this problem not only on women mortality and morbidity but also on the healthcare system a screening programs were introduced. One of the first screening programs were in united states which were introduced in the 1960s. ${ }^{(20)}$ The Papanicolaou test (pap smear) was first developed by DR George Papanicolaou in 1928 but not acknowledge until a research paper he published in 1943 with a colleague. ${ }^{(21)}$ Either conventional; or liquid-based Pap tests are acceptable for cervical cancer screening. ${ }^{(22)}$ Another screening modality is HPV testing either alone or in combination with Pap test which has more sensitivity than Pap smear alone. ${ }^{(23)}$ Cervical cancer screening with Pap smear and HPV testing acquired 100 percent sensitivity. ${ }^{(24)}$ As a result of the screening program, a dramatic drop were noticed in both mortality and morbidity especially for women above $40 .{ }^{(25,26)}$ 
Several guidelines were developed for cervical cancer screening according to their age and risk. ${ }^{(20,27)}$ There are different screening recommendations for patient who are immunocompromised, women with prior hysterectomy, recipients of the HPV vaccine, Diethylstilbestrol exposed women and women with prior History of CIN 2, CIN 3, or adenocarcinoma in situ, those patients have $\mathrm{e}^{(20,28,29)}$

Another effort to decrease this problem were made by the development of HPV vaccine. The efficacy of the vaccines were established by decreasing the rate of high-grade cervical neoplasia related to HPV-16 or HPV-18. ${ }^{(30)}$

To get the benefits from all these medical advances, appropriate education must be provided for health care workers and the public. This information must be provided for women to encourage the health-seeking behavior. Despite the important part that physicians play in cervical cancer prevention and education, research on this subject is limited concerning the current Saudi Arabian health care providers.

Furthermore, there is a lack of a national program in Saudi Arabia, which may be contributed to lower incidence of cervical cancer compared to the worldwide incidence. However, the limited available studies from Saudi Arabia indicates that HPV prevalence and genotypes' distribution in invasive cervical cancer shows a comparable pattern as in the world which indicate that we need a national screening program as some of the studies suggested that HPV prevalence is increasing ${ }^{(4)}$ This research is sought to explore the knowledge of PHC physicians regarding cervical cancer screening in Saudi Arabia that might help in improving the practice and in increasing the awareness.

This study aimed to estimates the level of knowledge of cervical cancer, cervical screening and to identify their determinants among MOH PHC physicians in Makkah Al-Mokarramah city 2016.

\section{Methodology:-}

\section{Study design:-}

A cross-sectional study was conduct among primary health care physicians working in Ministry of Health PHCCs in the three supervisory sectors inside Makkah AL-Mokarramah city.

The sample size was calculated by Raosoft Website for sample size calculation. It was 151 physicians, based on $50 \%$ prevalence, $95 \%$ confidence level, $5 \%$ error and $10 \%$ for defaulter and non-respondent.

Based on a through literature review, A self-administered questionnaire constructed by the researcher and was validated by three consultants. The questionnaire consisted of two main parts:

- First part: socio-demographic and personal characteristics including age, gender, medical degree, nationality, years of experience, the percentage of female patients monthly, referring patients for Pap test, reading about cervical cancer screening guidelines and last CME hours attended about cervical cancer.

- Second part: which consists of two domains

- Domain 1: It assess physicians' knowledge about cervical cancer, i.e., general knowledge about cervical cancer (6 questions), risk factors for cervical cancer (8 questions), symptoms of cervical cancer (4 questions), Staging of cervical cancer (5 questions), Treatment of cervical cancer (3 questions).The physicians' responses to these questions were either: true, false or Don't know

- Domain 2: It assess physicians' knowledge about cervical cancer screening guidelines, and it consists of 12 questions in which they have to answer with either: true, false or Don't know

Collected data were entered into an own computer and were analyzed using the SPSS version 21 with a significance of p-value $<0.05$. Each correct response of statements included in both domains was assigned a score of " 1 ", while incorrect response (or Don't know response) was assigned a score of "0". Therefore, the maximum score for Domain 1 was 26 while that for Domain 2 was 12, and the maximum total knowledge score was 38. Score percentage was computed. Participants who obtained $60 \%$ or more of the total score were considered as "knowledgeable" while those who obtained less than $60 \%$ of the total scores were considered as "Not knowledgeable".

Data were presented in the form of frequency and percentage. Pearson`s/likelihood ratio chi-square test was used to test for the association between different factors and knowledge of cancer cervix and its screening. Student-t-test was used to test for the difference between means of continuous variables (age, years of experience) in two different groups (knowledgeable and not knowledgeable). A P-value less than 0.05 was considered statistically significant. 
Permission from both the Joint Program of Family Medicine and from the general directorate of health affairsgeneral health section in Makkah Al-Mukarramah were obtained. A written consent (on the front page of the questionnaire) was obtained from each physician.

\section{Results:-}

The study included 137 physicians out of 151 invited to participate in the study with a response rate of $90.7 \%$.

Table (1) shows that the age of participants ranged from 25 to 60 years, with a mean of 39.7 years and a standard deviation of 8.8 years. Almost half of participants (46.7\%) were males, while $43.1 \%$ were Saudi. Most participants (85.4\%) were ever-married. Almost three-fourths of participants (70.1\%) were MBBS qualified, $19.7 \%$ were Boardcertified, 5.8\% were Diploma-certified, while $4.4 \%$ had other qualifications (e.g., Master Degree in surgery, MRCGP, Master in public health, diploma in obstetrics and gynecology). (Table 1)

Table (2) shows that 36.5\% of PHCCs were JCI/CBAH-accredited. Periodic health examination was stated by 43 participants that it is being conducted PHCCs (31.4\%). Pap smear testing was being applied by $46.5 \%$ of those who conduct periodic health examination. About one-third of PHCCs (33.6\%) receive 126 patients or more daily, for which the range of female patients seen at PHCCs ranges from 0 to 100 , with a mean of 48.3 and a standard deviation of 38.9. Almost half of PHCCs (47.4\%) are served by 4-6 PHC physicians, while $30.7 \%$ are served by 1-3 physicians, and $21.9 \%$ are served by 7 or more physicians. The type of medical records used by more than half of PHCCs (54\%) were paper files. (Table 2)

Table (3) shows that most participants (89.1\%) heard about Pap smear testing, while only $36.5 \%$ of participants had an MOH-protocol for cervical cancer screening. Among those who had an MOH-protocol of cervical cancer screening, 22 (44\%) had access to that protocol. About one-third of participants (33.6\%) ask asymptomatic female patients about screening for cancer cervix, while $14.6 \%$ always refer female patients for screening for cancer cervix, $16.8 \%$ do that sometimes, $18.2 \%$ rarely do it, and $50.4 \%$ never did that. About half of participants (49.6\%) read about cervical screening guidelines last year. Among those who read about cervical cancer screening guidelines, their main sources were medical textbooks $(66.2 \%)$ or online sources $(25 \%)$, while symposiums and lectures constituted only $8.8 \%$. About one fourth of participants (26.3\%) attended CME on cervical cancer and its screening last year. (Table 3)

Table (4) shows that the most frequently correctly answered statements by PHC physicians about cancer cervix were related to cervical cancer is preventable (78.8\%) and history of sexually transmitted infections as a high-risk factor for cervical cancer (73.7\%). On the other hand, the least frequently correctly answered statements were smoking and poor hygiene as risk factors for cervical cancer (20.4\% and $27.7 \%$, respectively). (Table 4 )

Table (5) shows that the most frequently correctly answered statements by PHC physicians about cancer cervix screening guidelines were related to "an 18 years old single female should not have Pap test (46.7\%) and "frequency of testing in average risk female is every three years" (48.9\%). On the other hand, the least frequently correctly answered statements were "the only screening method is Pap test" (12.4\%) and "a 30 years old female can be screened by Pap test with HPV testing every five years" (25.5\%). (Table 5)

Table (6) and figures (1-3) show that $42.3 \%$ of participants were knowledgeable regarding cancer cervix (Mean+SD were $54.1+20.3 \%$, while confidence intervals for percent knowledge scores were $50.7-57.5 \%$ ), $16.1 \%$ were knowledgeable regarding cancer cervix screening guidelines (Mean+SD were $34.6+27.9 \%$, while confidence intervals for percent knowledge scores were 29.9-39.3\%) and 31.4\% were knowledgeable regarding overall knowledge (Mean+SD were 47.9+19.4\%, while confidence intervals for percent knowledge scores were 44.7$51.2 \%$. (Table 6)

Table (7) shows that, regarding participants' knowledge about cancer cervix, Saudi PHC physicians were significantly less knowledgeable than non-Saudi physicians (30.5\% and $51.3 \%$, respectively $\mathrm{p}=0.015)$. Moreover, Board certified and Diploma certified PHC physicians were more knowledgeable than PHC physicians with other qualifications $(66.7 \%$ and $50 \%$, respectively, $\mathrm{p}=0.032)$. However, PHC physicians' knowledge grades regarding cancer cervix did not differ significantly according to participants' age groups, gender, marital status or their duration of experience in PHC. (Table 7) 
Table (8) shows that, regarding overall PHC physicians' knowledge about cancer cervix, the percentage of knowledgeable physicians at JCI/CBAH-accredited PHCCs was significantly higher than that at other PHCCs (52\% and $19.5 \%$, respectively, $\mathrm{p}<0.001$ ). Similarly, the percentage of knowledgeable physicians at PHCCs which perform periodic health examination was significantly higher than that at other PHCCs $(58.1 \%$ and $20.5 \%$, respectively, $\mathrm{p}<0.001)$. Moreover, the percentage of knowledgeable physicians at PHCCs which receive higher numbers of patients (>126/day) was significantly highest (50\%), compared with those at other PHCCs ( $\mathrm{p}=0.010)$. In addition, the percentage of knowledgeable physicians at PHCCs which have higher numbers of physicians (>7) was significantly highest $(56.7 \%)$, compared with those at other PHCCs $(\mathrm{p}<0.001)$. However, the percentage of knowledgeable PHCC physicians did not differ significantly according to the inclusion of Pap-smear, the percent of female patients or type of medical records at PHCC. (Table 8)

Table (9) shows that, regarding PHC physicians' knowledge about cancer cervix, the percentage of knowledgeable physicians who heard about Pap smear was significantly more knowledgeable than those who did not hear about it (47.5\% and $0 \%$, respectively, $\mathrm{p}<0.001$ ). Moreover, the percentage of knowledgeable physicians who attended CME on cervical cancer and its screening was more knowledgeable than those who did not attended it $(61.1 \%$ and $36.1 \%$, respectively, $\mathrm{p}=0.027$. However, the percentage of knowledgeable PHCC physicians did not differ significantly according to having an $\mathrm{MOH}$-protocol of cervical cancer screening, access to $\mathrm{MOH}$-protocol of cervical cancer screening, asking asymptomatic patients about cancer cervix screening, referring asymptomatic patients for cancer cervix screening, reading last year about cervical screening guidelines or sources of reading about cervical screening guidelines. (Table 9)

Table (7) shows that, regarding participants' knowledge about cancer cervix screening guidelines, PHC physicians' knowledge grades did not differ significantly according to their personal characteristics. (Table 7)

Table (8) shows that, regarding PHC physicians' knowledge about cancer cervix screening guidelines, the percentage of knowledgeable physicians did not differ significantly according to their PHCC characteristics. (Table 8)

Table (9) shows that, regarding PHC physicians' knowledge about cancer cervix screening guidelines, percentages of knowledgeable physicians who had their reading sources from online sources $(41.2 \%)$ was significantly higher than those who read from other sources ( $\mathrm{p}=0.013$ ). However, percentage of knowledgeable PHCC physicians did not differ significantly according to hearing about Pap smear, having a MOH-protocol of cervical cancer screening, access for $\mathrm{MOH}$-protocol of cervical cancer screening, asking asymptomatic patients about cancer cervix screening, referring asymptomatic patients for cancer cervix screening, reading last year about cervical screening guidelines, or attending last year CME on cervical cancer or its screening. (Table 9)

Table (7) shows that, regarding participants' overall knowledge about cancer cervix, Saudi PHC physicians were significantly less knowledgeable than non-Saudi physicians $(22 \%$ and $38.5 \%$, respectively $\mathrm{p}=0.040)$. Moreover, Board certified PHC physicians were more knowledgeable than PHC physicians with other qualifications (51.9\%, $\mathrm{p}=0.035$ ). However, PHC physicians' overall knowledge grades regarding cancer cervix did not differ significantly according to participants' age groups, gender, marital status or their duration of experience in PHC. (Table 7)

Table (8) shows that, regarding overall PHC physicians' knowledge about cancer cervix, the percentage of knowledgeable physicians at JCI/CBAH-accredited PHCCs was significantly higher than that at other PHCCs (52\% and $19.5 \%$, respectively, $\mathrm{p}<0.001$ ). Similarly, the percentage of knowledgeable physicians at PHCCs which perform periodic health examination was significantly higher than that at other PHCCs $(58.1 \%$ and $20.5 \%$, respectively, $\mathrm{p}<0.001)$. Moreover, the percentage of knowledgeable physicians at PHCCs which receive higher numbers of patients (>126/day) was significantly highest (50\%), compared with those at other PHCCs $(\mathrm{p}=0.010)$. In addition, the percentage of knowledgeable physicians at PHCCs which have higher numbers of physicians (>7) was significantly highest $(56.7 \%)$, compared with those at other PHCCs $(\mathrm{p}<0.001)$. However, the percentage of knowledgeable PHCC physicians did not differ significantly according to the inclusion of Pap-smear, percent of female patients or type of medical records at PHCC. (Table 8)

Table (9) shows that, regarding PHC physicians' knowledge about cancer cervix screening guidelines, the percentage of knowledgeable physicians who heard about Pap smear was significantly higher than those who did not 
hear about it $(35.2 \%$ and $0 \%$, respectively, $\mathrm{p}=0.006)$. The percentage of knowledgeable physicians who ask asymptomatic patients about cancer cervix screening was significantly higher than those who do not ask about it (45.7\% and $24.2 \%$, respectively, $\mathrm{p}=0.011)$. Moreover, the percentage of knowledgeable physicians who read online sources about cancer cervix $(58.8 \%)$ was significantly higher than those who read from other sources $(\mathrm{p}=0.032)$. In addition, percentage of knowledgeable physicians who attended CME on cervical cancer and its screening was significantly higher than those who did not attend (50\% and $25.8 \%$, respectively, $\mathrm{p}=0.007)$. However, the percentage of knowledgeable PHCC physicians did not differ significantly according to having an MOH-protocol of cervical cancer screening, access to $\mathrm{MOH}$-protocol of cervical cancer screening, referring asymptomatic patients for cancer cervix screening, or reading last year about cervical screening guidelines. (Table 9)

Table 1:- Personal characteristics of study sample $(n=137)$

\begin{tabular}{|c|l|}
\hline Personal characteristics & Value \\
\hline Age groups (in years) & \\
\hline$\bullet \quad$ Range & $25-60$ \\
\hline$\bullet \quad$ Mean+SD & $39.7+8.8$ \\
\hline Gender & \\
\hline$\bullet \quad$ Male & $64(46.7 \%)$ \\
\hline Nationality & $73(53.3 \%)$ \\
\hline$\bullet \quad$ Saudi & \\
\hline$\bullet \quad$ Non-Saudi & $59(43.1 \%)$ \\
\hline Marital status & $78(56.9 \%)$ \\
\hline$\bullet \quad$ Ever married & \\
\hline$\bullet \quad$ Never married & $117(85.4 \%)$ \\
\hline Qualification & $20(14.6 \%)$ \\
\hline$\bullet \quad$ MBBS & \\
\hline$\bullet \quad$ Board certified & $96(70.1 \%)$ \\
\hline$\bullet \quad$ Other & $27(19.7 \%)$ \\
\hline Years of experience & $8(5.8 \%)$ \\
\hline$\bullet \quad$ Range & $6(4.4 \%)$ \\
\hline$\bullet \quad$ Mean+SD & \\
\hline
\end{tabular}

Table 2:- Characteristics of participants' primary health care centers

\begin{tabular}{|c|l|}
\hline PHCC characteristics & Value \\
\hline JCI/CBAH-accredited PHCC & $50(36.5 \%)$ \\
\hline$\bullet \quad$ Yes & $43(31.4 \%)$ \\
\hline Periodic health examination at PHCC & $20(46.5 \%)$ \\
\hline No. of patients seen at PHCC & $20(14.6 \%)$ \\
\hline$\bullet \quad<25$ & $31(22.6 \%)$ \\
\hline$\bullet 26-50$ & $18(13.1 \%)$ \\
\hline$\bullet 51-75$ & $11(8.0 \%)$ \\
\hline$\bullet 76-100$ & $11(8.0 \%)$ \\
\hline$\bullet \quad>126$ & $46(33.6 \%)$ \\
\hline$\bullet \quad 1-3$ & \\
\hline$\bullet \quad 4-6$ & $42(30.7 \%)$ \\
\hline$\bullet 7$ or more & $65(47.4 \%)$ \\
\hline Type of medical records at PHCC & $30(21.9 \%)$ \\
\hline
\end{tabular}




\begin{tabular}{|c|l|}
\hline$\bullet \quad$ paper files & $74(54.0 \%)$ \\
\hline$\bullet \quad$ prescriptions only & $63(46.0 \%)$ \\
\hline Percentage of female patients at PHCC & $0-100$ \\
\hline$\bullet \quad$ Range & $48.3+38.9$ \\
\hline$\bullet \quad$ Mean+SD & 00 \\
\hline
\end{tabular}

Table 3:- Primary health care physicians' awareness and practices regarding cervical cancer screening

\begin{tabular}{|l|l|l|}
\hline Variables & No. & $\%$ \\
\hline Hearing about Pap smear & 122 & 89.1 \\
\hline Having an MOH-protocol of cervical cancer screening & 50 & 36.5 \\
\hline Access to MOH-protocol of cervical cancer screening (n=50) & 22 & 44.0 \\
\hline Asking asymptomatic females about screening for cancer cervix & 46 & 33.6 \\
\hline Referring asymptomatic females for cancer cervix screening & & \\
\hline$\bullet \quad$ Always & 20 & 14.6 \\
\hline$\bullet \quad$ Sometimes & 23 & 16.8 \\
\hline$\bullet \quad$ Rarely & 25 & 18.2 \\
\hline$\bullet \quad$ Never & 69 & 50.4 \\
\hline Reading about cervical screening guidelines during last year & 68 & 49.6 \\
\hline Sources of reading about cervical screening guidelines (n=68) & & \\
\hline$\bullet \quad$ Medical textbooks & 45 & 66.2 \\
\hline$\bullet \quad$ Symposiums/lectures & 6 & 8.8 \\
\hline$\bullet \quad$ Online & 17 & 25.0 \\
\hline Attending CME on cervical cancer or its screening during last year & 36 & 26.3 \\
\hline
\end{tabular}

Table (4):- Primary health care physicians' correct responses regarding cancer cervix

Knowledge items

Cervical cancer is one of the top 10 malignancies in Saudi females

Cervical cancer is preventable

HPV is fundamental to the development of cervical neoplasia

The most common site for cervical cancer is ectocervix

CIN is a precancerous lesion of cervix

Cervical cancer occurs many years after CIN

High risk factors for cervical cancer includes:

- Early sexual debut

- Early age of first pregnancy

- Multiple sexual partners

- Multiple births

- Oral contraceptive pills

- Smoking

- Poor genital hygiene

- History of sexually transmitted infections

Common symptoms of cervical cancer

- Offensive foul-smelling discharge

- Postmenopausal bleeding

- Post-coital bleeding

- Irregular bleeding

Staging of cervical cancer can be done by:

- Clinical examination

- Biopsy

- Colposcopy

- Imaging

- Surgical

\begin{tabular}{|l|l|}
\hline No. & $\%$ \\
\hline 82 & 59.9 \\
\hline 108 & 78.8 \\
\hline 92 & 67.2 \\
\hline 68 & 49.6 \\
\hline 80 & 58.4 \\
\hline 66 & 48.2 \\
\hline & \\
\hline 84 & 61.3 \\
\hline 71 & 51.8 \\
\hline 89 & 65.0 \\
\hline 61 & 44.5 \\
\hline 70 & 51.1 \\
\hline 28 & 20.4 \\
\hline 38 & 27.7 \\
\hline 101 & 73.7 \\
\hline & \\
\hline 71 & 51.8 \\
\hline 86 & 62.8 \\
\hline 84 & 61.3 \\
\hline 73 & 53.3 \\
\hline & \\
\hline 42 & 30.7 \\
\hline 92 & 67.2 \\
\hline 62 & 45.3 \\
\hline 62 & 45.3 \\
\hline 69 & 50.4 \\
\hline & \\
\hline
\end{tabular}




\begin{tabular}{|c|c|c|}
\hline \multicolumn{2}{|l|}{ Treatment of cervical cancer includes: } & \\
\hline$\bullet \quad$ Radiotherapy & 75 & 54.7 \\
\hline$\bullet \quad$ Chemotherapy & 82 & 59.9 \\
\hline$\bullet \quad$ Surgery & 91 & 66.4 \\
\hline
\end{tabular}

Table (5):- Primary health care physicians' correct responses regarding guidelines for cancer cervix screening

\begin{tabular}{|c|c|c|}
\hline Knowledge items & No. & $\%$ \\
\hline The only screening method is Pap test (False) & 17 & 12.4 \\
\hline Screening is unsafe and its harmful effect outweighs its benefit (False) & 56 & 40.9 \\
\hline The initiation of cervical screening starts at the age of 21 years (True) & 62 & 45.3 \\
\hline Discontinuation age of cervical cancer screening is at 80 years (False) & 50 & 36.5 \\
\hline Frequency of testing in average risk female is every three years (True) & 67 & 48.9 \\
\hline An 18 years old single female should not have Pap test (True) & 64 & 46.7 \\
\hline $\begin{array}{l}\text { A } 25 \text { years old married female with three consecutive negative results can stop performing Pap } \\
\text { test (False) }\end{array}$ & 38 & 27.7 \\
\hline A 30 years old female can be screened by Pap test with HPV testing every five years (True) & 35 & 25.5 \\
\hline A 30 years old female can be screened by Pap test every three years (True) & 59 & 43.1 \\
\hline A 27 years old female who received HPV vaccine should discontinue screening (False) & 38 & 27.7 \\
\hline $\begin{array}{l}\text { A } 66 \text { years old female with three consecutive negative Pap tests in the past ten years should } \\
\text { discontinue screening (True) }\end{array}$ & 51 & 37.2 \\
\hline A 65 years old smoker female should undergo screening at regular intervals (True) & 49 & 35.8 \\
\hline
\end{tabular}

Table (6):- Distribution of participants' knowledge grades, Mean+SD and confidence intervals for percent knowledge scores according to their knowledge domains

\begin{tabular}{|c|c|c|c|c|c|c|}
\hline Knowledge & Not & ledgeable & Kno & eable & Percent & Confidence \\
\hline Domains & No. & $\%$ & No. & $\%$ & Mean+SD & Intervals \\
\hline Cancer cervix & 79 & 57.7 & 58 & 42.3 & $54.1+20.3$ & $50.7-57.5 \%$ \\
\hline Screening guidelines & 115 & 83.9 & 22 & 16.1 & $34.6+27.9$ & $29.9-39.3 \%$ \\
\hline Overall knowledge & 94 & 68.6 & 43 & 31.4 & $47.9+19.4$ & $44.7-51.2 \%$ \\
\hline
\end{tabular}

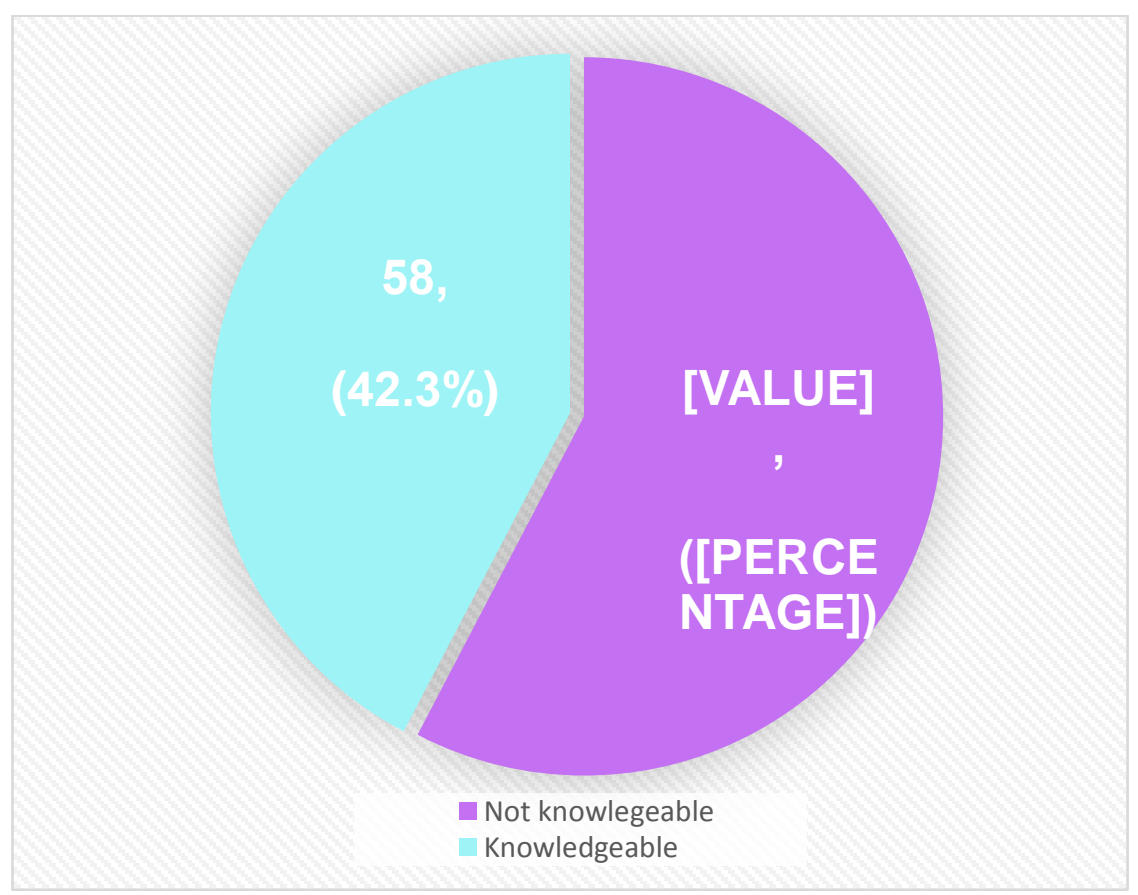

Figure 1:- Knowledge level regarding cervical cancer among physicians. 


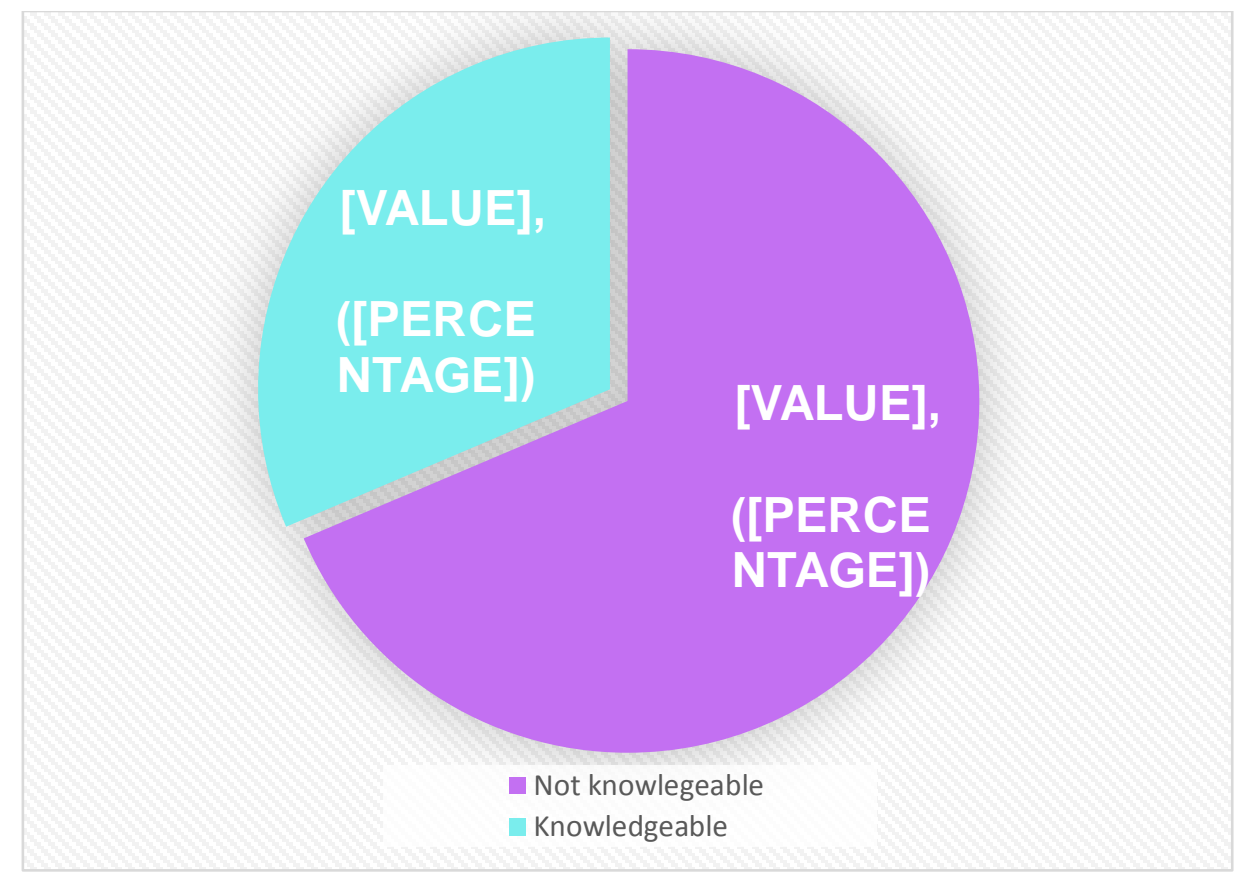

Figure 3:- Overall knowledge level regarding cervical cancer among physicians.

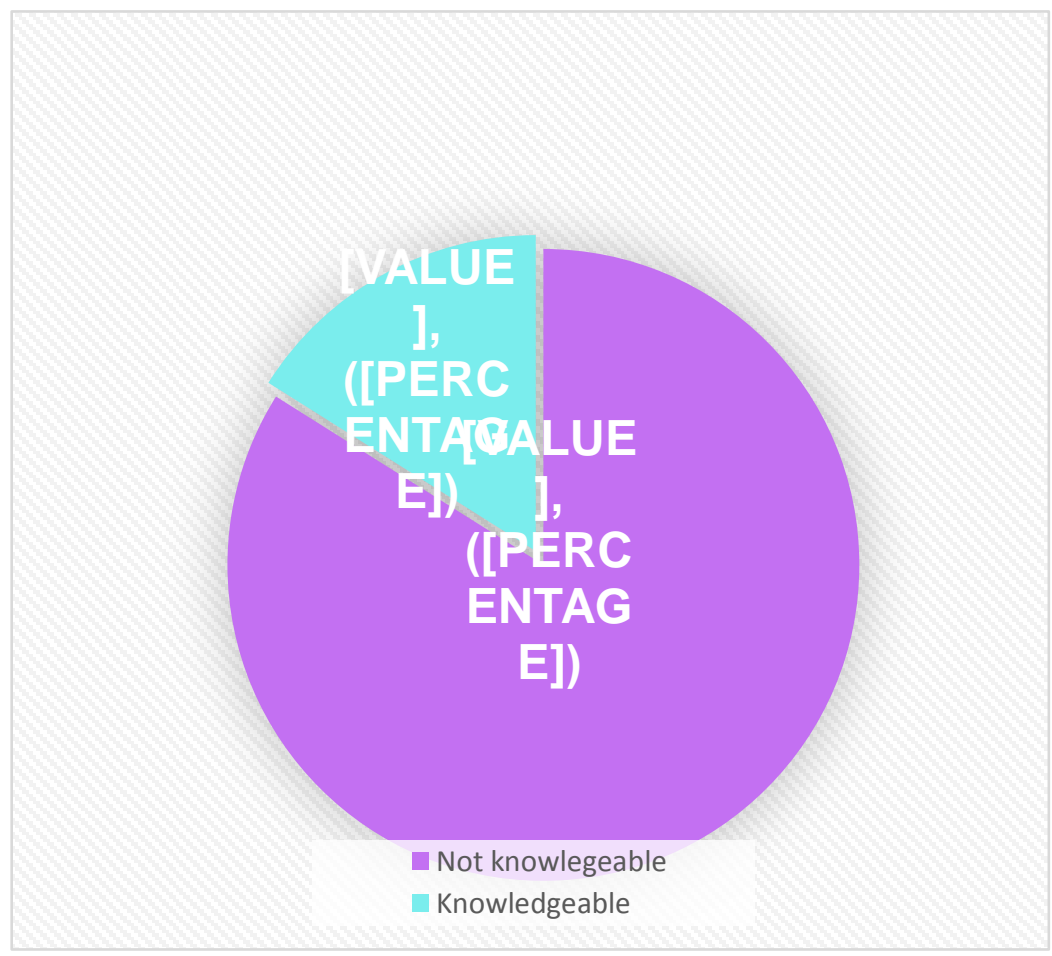

Figure 2:- Knowledge level regarding cervical cancer screening guidelines among physicians. 
Table (7):- Primary health care physicians' knowledge grades about cancer cervix, cancer cervix screening guidelines and overall knowledge according to their personal characteristics

\begin{tabular}{|c|c|c|c|c|c|c|}
\hline \multirow[t]{2}{*}{$\begin{array}{l}\text { Personal } \\
\text { Characteristics }\end{array}$} & \multicolumn{2}{|c|}{$\begin{array}{l}\text { general knowledge level } \\
\text { about cancer cervix }\end{array}$} & \multicolumn{2}{|c|}{$\begin{array}{l}\text { knowledge level about } \\
\text { cancer cervix screening } \\
\text { guidelines }\end{array}$} & \multicolumn{2}{|c|}{ overall knowledge } \\
\hline & $\begin{array}{l}\text { Not } \\
\text { Knowledgea } \\
\text { ble }(\mathrm{n}=79)\end{array}$ & $\begin{array}{l}\text { Knowledgea } \\
\text { ble } \\
(\mathrm{n}=58)\end{array}$ & $\begin{array}{l}\text { Not } \\
\text { Knowledgea } \\
\text { ble }(n=115)\end{array}$ & $\begin{array}{l}\text { Knowledgea } \\
\text { ble } \\
(\mathrm{n}=22)\end{array}$ & $\begin{array}{l}\text { Not } \\
\text { Knowledgea } \\
\text { ble }(n=94)\end{array}$ & $\begin{array}{l}\text { Knowledgea } \\
\text { ble } \\
(n=43)\end{array}$ \\
\hline \multicolumn{7}{|l|}{$\begin{array}{l}\text { Age groups (in } \\
\text { years) }\end{array}$} \\
\hline \multirow[t]{2}{*}{ - $\quad$ Mean \pm SD } & $39.1 \pm 8.8$ & $40.4 \pm 8.7$ & $39.4 \pm 8.5$ & $40.8 \pm 10.1$ & $38.7 \pm 8.6$ & $41.7 \pm 8.9$ \\
\hline & \multicolumn{2}{|c|}{$\mathrm{t}=0.906 \mathrm{P}=0.367$} & \multicolumn{2}{|c|}{$\mathrm{t}=0.678 \quad \mathrm{P}=0.499$} & \multicolumn{2}{|c|}{$\mathrm{t}=1.841 \quad \mathrm{P}=0.068$} \\
\hline \multicolumn{7}{|l|}{ Gender } \\
\hline - Male & $36(56.3 \%)$ & $28(43.8 \%)$ & \multirow{2}{*}{$\begin{array}{l}54(84.4 \%) \\
61(83.6 \%)\end{array}$} & $10(15.6 \%)$ & $40(62.5 \%)$ & $24(37.5 \%)$ \\
\hline \multirow[t]{2}{*}{ - Female } & $43(58.9 \%)$ & $30(41.1 \%)$ & & $12(16.4 \%)$ & $54(74.0 \%)$ & $19(26.0 \%)$ \\
\hline & \multicolumn{2}{|c|}{$\chi^{2}=0.098 \quad \mathrm{P}=0.754$} & \multicolumn{2}{|c|}{$\chi^{2}=0.017 \quad \mathrm{P}=0.897$} & \multicolumn{2}{|c|}{$\chi^{2}=2.084 \quad \mathrm{P}=0.149$} \\
\hline \multicolumn{7}{|l|}{ Nationality } \\
\hline - Saudi & $41(69.5 \%)$ & $18(30.5 \%)$ & \multirow{2}{*}{$\begin{array}{l}49(83.1 \%) \\
66(84.6 \%)\end{array}$} & $10(16.9 \%)$ & $46(78.0 \%)$ & $13(22.0 \%)$ \\
\hline \multirow[t]{2}{*}{ - Non-Saudi } & $38(48.7 \%)$ & $40(51.3 \%)$ & & $12(15.4 \%)$ & $48(61.5 \%)$ & $30(38.5 \%)$ \\
\hline & \multicolumn{2}{|c|}{$\chi^{2}=5.938=0.015$} & \multicolumn{2}{|c|}{$\chi^{2}=0.061 \quad \mathrm{P}=0.805$} & \multicolumn{2}{|c|}{$\chi^{2}=4.209 \quad \mathrm{P}=0.040$} \\
\hline \multicolumn{7}{|l|}{ Marital status } \\
\hline - Ever married & $66(56.4 \%)$ & $51(43.6 \%)$ & \multirow{2}{*}{$\begin{array}{l}98(83.8 \%) \\
17(85.0 \%)\end{array}$} & $19(16.2 \%)$ & $80(68.4 \%)$ & $37(31.6 \%)$ \\
\hline \multirow[t]{2}{*}{ - Never married } & $13(65.0 \%)$ & $7(35.0 \%)$ & & $3(15.0 \%)$ & $14(70.0 \%)$ & $6(30.0 \%)$ \\
\hline & \multicolumn{2}{|c|}{$\chi^{2}=0.516 \quad P=0.472$} & \multicolumn{2}{|c|}{$\chi^{2}=0.019 \quad \mathrm{P}=0.889$} & \multicolumn{2}{|c|}{$\chi^{2}=0.021 \quad \mathrm{P}=0.885$} \\
\hline \multicolumn{7}{|l|}{ Qualification } \\
\hline - MBBS & $62(64.6 \%)$ & $34(35.4 \%)$ & $84(87.5 \%)$ & $12(12.5 \%)$ & $71(74.0 \%)$ & $25(26.0 \%)$ \\
\hline - Board certified & $9(33.3 \%)$ & $18(66.7 \%)$ & \multirow{2}{*}{$\begin{array}{l}19(70.4 \%) \\
8(100.0 \%)\end{array}$} & $8(29.6 \%)$ & $13(48.1 \%)$ & $14(51.9 \%)$ \\
\hline $\begin{array}{ll} & \text { Diploma } \\
& \text { certified }\end{array}$ & $4(50.0 \%)$ & $4(50.0 \%)$ & & $0(0.0 \%)$ & $7(87.5 \%)$ & $1(12.5 \%)$ \\
\hline \multirow[t]{2}{*}{ - Other } & $4(66.7 \%)$ & $2(33.3 \%)$ & $4(66.7 \%)$ & $2(33.3 \%)$ & $3(50.0 \%)$ & $3(50.0 \%)$ \\
\hline & \multicolumn{2}{|c|}{$\chi^{2}=8.793^{(1)} P=0.032$} & \multicolumn{2}{|c|}{$\chi^{2}=7.45^{(1)} \quad \mathrm{P}=0.059$} & \multicolumn{2}{|c|}{$\chi^{2}=8.62^{(1)} \mathrm{P}=0.035$} \\
\hline $\begin{array}{l}\text { Years of experience } \\
\text { in PHC }\end{array}$ & & & & & & \\
\hline - Mean \pm SD & $11.9 \pm 8.2$ & $13.2 \pm 8.1$ & $11.9 \pm 8.2$ & $13.2 \pm 8.1$ & $11.6 \pm 8.1$ & $14.2 \pm 8.1$ \\
\hline & $\mathrm{t}=0.918 \quad \mathrm{P}=0$. & & $\mathrm{t}=0.537 \quad \mathrm{P}=$ & .592 & $\mathrm{t}=1.733 \mathrm{P}=0$ & \\
\hline
\end{tabular}

Table (8):- Primary health care physicians' general knowledge level about cancer cervix according, cancer cervix screening guidelines and overall knowledge to PHCC characteristics

\begin{tabular}{|c|c|c|c|c|c|c|}
\hline \multirow[t]{2}{*}{$\begin{array}{l}\text { PHCC } \\
\text { characteristics }\end{array}$} & \multicolumn{2}{|c|}{$\begin{array}{l}\text { general knowledge level } \\
\text { about cancer cervix }\end{array}$} & \multicolumn{2}{|c|}{$\begin{array}{l}\text { knowledge level about } \\
\text { cancer cervix screening } \\
\text { guidelines }\end{array}$} & \multicolumn{2}{|c|}{ overall knowledge } \\
\hline & $\begin{array}{l}\text { Not } \\
\text { Knowledgea } \\
\text { ble }(\mathrm{n}=79)\end{array}$ & $\begin{array}{l}\text { Knowledgea } \\
\text { ble } \\
(\mathrm{n}=58)\end{array}$ & $\begin{array}{l}\text { Not } \\
\text { Knowledgea } \\
\text { ble }(\mathrm{n}=115)\end{array}$ & $\begin{array}{l}\text { Knowledgea } \\
\text { ble } \\
(\mathrm{n}=22)\end{array}$ & $\begin{array}{l}\text { Not } \\
\text { Knowledgea } \\
\text { ble }(\mathrm{n}=94)\end{array}$ & $\begin{array}{l}\text { Knowledgea } \\
\text { ble } \\
(\mathrm{n}=43)\end{array}$ \\
\hline \multicolumn{7}{|l|}{$\begin{array}{l}\text { JCI/CBAH- } \\
\text { accredited PHCC }\end{array}$} \\
\hline - Yes & $17(34.0 \%)$ & $33(66.0 \%)$ & $40(80.0 \%)$ & $10(20.0 \%)$ & $24(48.0 \%)$ & $26(52.0 \%)$ \\
\hline - $\mathrm{No}$ & $62(71.3 \%)$ & $25(28.7 \%)$ & $75(86.2 \%)$ & $12(13.8 \%)$ & $70(80.5 \%)$ & $17(19.5 \%)$ \\
\hline & \multicolumn{2}{|c|}{$\chi^{2}=18.061 \quad \mathrm{P}<0.001$} & \multicolumn{2}{|c|}{$\chi^{2}=0.907 \quad \mathrm{P}=0.341$} & \multicolumn{2}{|c|}{$\chi^{2}=15.535 \quad \mathrm{P}<0.001$} \\
\hline $\begin{array}{lr}\text { Periodic } & \text { health } \\
\text { examination } & \text { at }\end{array}$ & & & & & & \\
\hline
\end{tabular}




\begin{tabular}{|c|c|c|c|c|c|c|}
\hline PHCC & & & & & & \\
\hline - Yes & $16(37.2 \% 0$ & $27(62.8 \%)$ & \multirow{2}{*}{$\begin{array}{l}34(79.1 \%) \\
72(86.7 \%)\end{array}$} & $9(20.9 \%)$ & $18(41.9 \%)$ & $25(58.1 \%)$ \\
\hline $\begin{array}{ll}- & \text { No }\end{array}$ & $53(63.9 \%)$ & $30(36.1 \%)$ & & $11(13.3 \%)$ & $66(79.5 \%)$ & $17(20.5 \%)$ \\
\hline \multirow{2}{*}{ - don't know } & $10(90.9 \%)$ & $1(9.1 \%)$ & $9(81.8 \%)$ & $2(18.2 \%)$ & $10(90.9 \%)$ & $1(9.1 \%)$ \\
\hline & \multicolumn{2}{|c|}{$\chi^{2}=15.61^{(1)} \mathrm{P}=0.001$} & \multicolumn{2}{|c|}{$\chi^{2}=1.279 \quad \mathrm{P}=0.528$} & \multicolumn{2}{|c|}{$\chi 2=21.413 \quad \mathrm{P}<0.001$} \\
\hline \multicolumn{7}{|l|}{$\begin{array}{l}\text { Inclusion of Pap- } \\
\text { testing }\end{array}$} \\
\hline - Yes & $4(20.0 \%)$ & $16(80.0 \%)$ & \multirow{2}{*}{$\begin{array}{l}19(95.0 \%) \\
13(65.0 \%)\end{array}$} & $1(5.0 \%)$ & $6(30.0 \%)$ & $14(70.0 \%)$ \\
\hline - $\quad \mathrm{No}$ & $12(60.0 \%)$ & $8(40.0 \%)$ & & $7(35.0 \%)$ & $11(55.0 \%)$ & $9(45.0 \%)$ \\
\hline \multirow[t]{2}{*}{ - don't know } & $0(0.0 \%)$ & $3(100.0 \%)$ & $2(66.7 \%)$ & $1(33.3 \%)$ & $1(33.3 \%)$ & $2(66.7 \%)$ \\
\hline & \multicolumn{2}{|c|}{$\chi^{2}=9.83^{(1)} \mathrm{P}=0.007$} & \multicolumn{2}{|c|}{$\chi^{2}=6.74^{(1)} \quad \mathrm{P}=0.057$} & \multicolumn{2}{|c|}{$\chi 2=2.69(1) \quad \mathrm{P}=0.261$} \\
\hline \multicolumn{7}{|l|}{$\begin{array}{l}\text { No. of patients seen } \\
\text { at PHCC }\end{array}$} \\
\hline$\bullet \quad<25$ & $14(70.0 \%)$ & $6(30.0 \%)$ & $20(100.0 \%)$ & $0(0.0 \%)$ & $17(85.0 \%)$ & $3(15.0 \%)$ \\
\hline - $\quad 26-50$ & $23(74.2 \%)$ & $8(25.8 \%)$ & $26(83.9 \%)$ & $5(16.1 \%)$ & $27(87.1 \%)$ & $4(12.9 \%)$ \\
\hline - $51-75$ & $12(66.7 \%)$ & $6(33.3 \%)$ & $13(72.2 \%)$ & $5(27.8 \%)$ & $12(66.7 \%)$ & $6(33.3 \%)$ \\
\hline - $76-100$ & $7(63.6 \%)$ & $4(36.4 \%)$ & $8(72.7 \%)$ & $3(27.3 \%)$ & $7(63.6 \%)$ & $4(36.4 \%)$ \\
\hline - $101-125$ & $8(72.7 \%)$ & $3(27.3 \%)$ & $10(90.9 \%)$ & $1(9.1 \%)$ & $8(72.7 \%)$ & $3(27.3 \%)$ \\
\hline \multirow[t]{2}{*}{ - $\quad>126$} & $15(32.6 \%)$ & $31(67.4 \%)$ & $38(82.6 \%)$ & $8(17.4 \%)$ & $23(50.0 \%)$ & $23(50.0 \%)$ \\
\hline & \multicolumn{2}{|c|}{$\chi^{2}=18.54^{(1)} \mathrm{P}=0.002$} & \multicolumn{2}{|c|}{$\chi^{2}=9.97^{(1)} \mathrm{P}=0.076$} & \multicolumn{2}{|c|}{$\chi 2=15.73(1) \quad \mathrm{P}=0.008$} \\
\hline \multicolumn{7}{|l|}{$\begin{array}{l}\text { Percent of females at } \\
\text { PHCC }\end{array}$} \\
\hline \multirow[t]{2}{*}{ - Mean \pm SD } & $42.8 \pm 39.9$ & $55.9 \pm 36.3$ & $48.4 \pm 39.0$ & $48.0 \pm 38.9$ & $46.4 \pm 39.5$ & $52.5 \pm 37.6$ \\
\hline & \multicolumn{2}{|c|}{$\mathrm{t}=1.971 \quad \mathrm{P}=0.051$} & \multicolumn{2}{|c|}{$\mathrm{t}=0.040 \quad \mathrm{P}=0.968$} & \multicolumn{2}{|c|}{$\mathrm{t}=0.851 \quad \mathrm{P}=0.396$} \\
\hline \multicolumn{7}{|l|}{$\begin{array}{l}\text { No. of physicians at } \\
\text { PHCC }\end{array}$} \\
\hline - $1-3$ & $32(76.2 \%)$ & $10(23.8 \%)$ & $38(90.5 \%)$ & $4(9.5 \%)$ & $37(88.1 \%)$ & $5(11.9 \%)$ \\
\hline - $4-6$ & $36(55.4 \%)$ & $29(44.6 \%)$ & $54(83.1 \%)$ & $11(16.9 \%)$ & $44(67.7 \%)$ & $21(32.3 \%)$ \\
\hline \multirow[t]{2}{*}{ - 7 or more } & $11(36.7 \%)$ & $19(63.3 \%)$ & $23(76.7 \%)$ & $7(23.3 \%)$ & $13(43.3 \%)$ & $17(56.7 \%)$ \\
\hline & \multicolumn{2}{|c|}{$\chi^{2}=11.461 \quad \mathrm{P}=0.003$} & \multicolumn{2}{|c|}{$\chi^{2}=2.455 \quad \mathrm{P}=0.280$} & \multicolumn{2}{|c|}{$\chi^{2}=16.330 \quad P<0.001$} \\
\hline $\begin{array}{l}\text { Type of medical } \\
\text { records at PHCC }\end{array}$ & & & $60(81.1 \%)$ & & & \\
\hline - $\quad$ Paper files & $44(59.5 \%)$ & $30(40.5 \%)$ & & $14(18.9 \%)$ & $52(70.3 \%)$ & $22(29.7 \%)$ \\
\hline \multirow[t]{2}{*}{$\begin{array}{ll}\text { - } & \begin{array}{l}\text { Prescriptions } \\
\text { only }\end{array} \\
\end{array}$} & $35(55.6 \%)$ & $28(44.4 \%)$ & $55(87.3 \%)$ & $8(12.7 \%)$ & $42(66.7 \%)$ & $21(33.3 \%)$ \\
\hline & \multicolumn{2}{|c|}{$\chi^{2}=0.212 \quad P=0.645$} & \multicolumn{2}{|c|}{$\chi^{2}=0.977 \mathrm{P}=0.226$} & \multicolumn{2}{|c|}{$\chi^{2}=0.205 \mathrm{P}=0.651$} \\
\hline
\end{tabular}

Table (9):- Primary health care physicians' general knowledge level about cancer cervix, cancer cervix screening guidelines and overall knowledge according to their practice

\begin{tabular}{|c|l|l|l|l|l|l|}
\hline Practices & \multicolumn{2}{|l|}{$\begin{array}{l}\text { General knowledge level } \\
\text { about cancer cervix }\end{array}$} & $\begin{array}{l}\text { Knowledge level about } \\
\text { cancer cervix screening }\end{array}$ & \multicolumn{2}{l|}{ Overall knowledge } \\
\cline { 2 - 7 } & $\begin{array}{l}\text { Not } \\
\text { Knowledge } \\
\text { able (n=79) }\end{array}$ & $\begin{array}{l}\text { Knowledge } \\
\text { able } \\
(\mathrm{n}=58)\end{array}$ & $\begin{array}{l}\text { Not } \\
\text { Knowledge } \\
\text { able } \\
(\mathrm{n}=115)\end{array}$ & $\begin{array}{l}\text { Knowledge } \\
\text { able } \\
(\mathrm{n}=22)\end{array}$ & $\begin{array}{l}\text { Not } \\
\text { Knowledge } \\
\text { able (n=94) }\end{array}$ & $\begin{array}{l}\text { Knowledge } \\
\text { able } \\
(\mathrm{n}=43)\end{array}$ \\
\hline Hearing about Pap smear & & & & & & \\
\hline$\bullet \quad$ Yes & $64(52.5 \%)$ & $58(47.5 \%)$ & $\begin{array}{l}101 \\
(82.8 \%)\end{array}$ & $21(17.2 \%)$ & $79(64.8 \%)$ & $43(35.2 \%)$ \\
\hline$\bullet \quad$ No & 15 & $0(0.0 \%)$ & $14(93.3 \%)$ & $1(6.7 \%)$ & $\begin{array}{l}15 \\
(100.0 \%)\end{array}$ & $0(0.0 \%)$ \\
\hline & $(100.0 \%)$ & & & $\chi^{2}=1.102 \quad \mathrm{P}=0.294$ & $\chi^{2}=7.705 \quad \mathrm{P}=0.006$ \\
\hline
\end{tabular}




\begin{tabular}{|c|c|c|c|c|c|c|}
\hline $\begin{array}{l}\text { Having an MOH-protocol } \\
\text { of cervical cancer screening }\end{array}$ & & & & & & \\
\hline$\bullet \quad$ Yes & $27(54.0 \%)$ & $23(46.0 \%)$ & \multirow{2}{*}{$\begin{array}{l}44(88.0 \%) \\
45(83.3 \%)\end{array}$} & $6(12.0 \%)$ & $30(60.0 \%)$ & $20(40.0 \%)$ \\
\hline - $\quad$ No & $32(59.3 \%)$ & $22(40.7 \%)$ & & $9(16.7 \%)$ & $38(70.4 \%)$ & $16(29.6 \%)$ \\
\hline \multirow[t]{2}{*}{ - $\quad$ don't know } & $20(60.6 \%)$ & $13(39.4 \%)$ & $26(78.8 \%)$ & $7(21.2 \%)$ & $26(78.8 \%)$ & $7(21.2 \%)$ \\
\hline & \multicolumn{2}{|c|}{$\chi^{2}=0.448 \quad \mathrm{P}=0.799$} & \multicolumn{2}{|c|}{$\chi^{2}=1.276 \quad \mathrm{P}=0.528$} & \multicolumn{2}{|c|}{$\chi^{2}=3.386 \quad \mathrm{P}=0.184$} \\
\hline \multicolumn{7}{|l|}{$\begin{array}{l}\text { Access to MOH-protocol of } \\
\text { cervical cancer screening }\end{array}$} \\
\hline$\bullet \quad$ Yes & $8(36.4 \%)$ & $14(63.6 \%)$ & \multirow{2}{*}{$\begin{array}{l}21(95.5 \%) \\
21(84.0 \%)\end{array}$} & $1(4.5 \%)$ & $10(45.5 \%)$ & $12(54.5 \%)$ \\
\hline - $\quad$ No & $17(68.0 \%)$ & $8(32.0 \%)$ & & $4(16.0 \%)$ & $18(72.0 \%)$ & $7(28.0 \%)$ \\
\hline \multirow[t]{2}{*}{ - $\quad$ don't know } & $2(66.7 \%)$ & $1(33.3 \%)$ & $2(66.7 \%)$ & $1(33.3 \%)$ & $2(66.7 \%)$ & $1(33.3 \%)$ \\
\hline & \multicolumn{2}{|c|}{$\chi^{2}=4.99^{(1)} \quad \mathrm{P}=0.082$} & \multicolumn{2}{|c|}{$\chi^{2}=2.75^{(1)} \quad \mathrm{P}=0.252$} & \multicolumn{2}{|c|}{$\chi^{2}=3.52^{(1)} \quad \mathrm{P}=0.172$} \\
\hline \multicolumn{7}{|l|}{$\begin{array}{l}\text { Asking asymptomatic } \\
\text { patients about cancer cervix } \\
\text { screening }\end{array}$} \\
\hline • Yes & $22(47.8 \%)$ & $24(52.2 \%)$ & \multirow{2}{*}{$\begin{array}{l}38(82.6 \%) \\
77(84.6 \%)\end{array}$} & $8(17.4 \%)$ & $25(54.3 \%)$ & $21(45.7 \%)$ \\
\hline \multirow[t]{2}{*}{ - $\quad$ No } & $57(62.6 \%)$ & $34(37.4 \%)$ & & $14(15.4 \%)$ & $69(75.8 \%)$ & $22(24.2 \%)$ \\
\hline & \multicolumn{2}{|c|}{$\chi^{2}=2.746 \quad \mathrm{P}=0.098$} & \multicolumn{2}{|c|}{$\chi^{2}=0.091 \quad \mathrm{P}=0.763$} & \multicolumn{2}{|c|}{$\chi^{2}=6.554 \quad \mathrm{P}=0.011$} \\
\hline \multicolumn{7}{|l|}{$\begin{array}{l}\text { Referring } \\
\text { patients for cancer cervix } \\
\text { screening }\end{array}$} \\
\hline$\bullet \quad$ Always & $9(45.0 \%)$ & $11(55.0 \%)$ & $\begin{array}{l}20 \\
(100.0 \%) \\
\end{array}$ & $0(0.0 \%)$ & $12(60.0 \%)$ & $8(40.0 \%)$ \\
\hline - $\quad$ Sometimes & $12(52.2 \%)$ & $11(47.8 \%)$ & $19(82.6 \%)$ & $4(17.4 \%)$ & $\begin{array}{l}13 \\
(56.5 \% 0\end{array}$ & $10(43.5 \%)$ \\
\hline - $\quad$ Rarely & $12(48.0 \%)$ & $13(52.0 \%)$ & $21(84.0 \%)$ & $4(16.0 \%)$ & $16(64.0 \%)$ & $9(36.0 \%)$ \\
\hline \multirow[t]{2}{*}{ - $\quad$ Never } & $46(66.7 \%)$ & $23(33.3 \%)$ & $55(79.7 \%)$ & $14(20.3 \%)$ & $53(76.8 \%)$ & $16(23.2 \%)$ \\
\hline & \multicolumn{2}{|c|}{$\chi^{2}=4.845 \quad \mathrm{P}=0.184$} & \multicolumn{2}{|c|}{$\chi^{2}=4.77^{(1)} \quad \mathrm{P}=0.189$} & \multicolumn{2}{|c|}{$\chi^{2}=4.651 \quad \mathrm{P}=0.199$} \\
\hline \multicolumn{7}{|l|}{$\begin{array}{l}\text { Reading last year about } \\
\text { cervical } \\
\text { guidelines }\end{array}$} \\
\hline - Yes & $39(57.4 \%)$ & $29(42.6 \%)$ & $55(80.9 \%)$ & $13(19.1 \%)$ & $44(64.7 \%)$ & $24(35.3 \%)$ \\
\hline - $\quad$ No & $33(61.1 \%)$ & $21(38.9 \%)$ & $46(85.2 \%)$ & $8(14.8 \%)$ & $40(74.1 \%)$ & $14(25.9 \%)$ \\
\hline \multirow[t]{2}{*}{ - don't remember } & $7(46.7 \%)$ & $8(53.3 \%)$ & $14(93.3 \%)$ & $1(6.7 \%)$ & $10(66.7 \%)$ & $5(33.3 \%)$ \\
\hline & \multicolumn{2}{|c|}{$\chi^{2}=1.009 \quad \mathrm{P}=0.604$} & \multicolumn{2}{|c|}{$\chi^{2}=1.516^{(1)} \quad \mathrm{P}=0.469$} & \multicolumn{2}{|c|}{$\chi^{2}=1.256 \quad \mathrm{P}=0.534$} \\
\hline \multicolumn{7}{|l|}{$\begin{array}{l}\text { Sources of reading about } \\
\text { cervical } \\
\text { guidelines }\end{array}$} \\
\hline • $\quad$ Medical books & $27(60.0 \%)$ & $18(40.0 \%)$ & $41(91.1 \%)$ & $4(8.9 \%)$ & $34(75.6 \%)$ & $11(24.4 \%)$ \\
\hline $\begin{array}{l}\text { - Symposiums/lect } \\
\text { ures }\end{array}$ & $4(66.7 \%)$ & $2(33.3 \%)$ & $4(66.7 \%)$ & $2(33.3 \%)$ & $3(50.0 \%)$ & $3(50.0 \%)$ \\
\hline - $\quad$ Online & $8(47.1 \%)$ & $9(52.9 \%)$ & $10(58.8 \%)$ & $7(41.2 \%)$ & $7(41.2 \%)$ & $10(58.8 \%)$ \\
\hline & $\chi^{2}=1.08^{(1)}$ & $=0.584$ & $\chi^{2}=8.69^{(1)} \mathrm{F}$ & $=0.013$ & $\chi^{2}=6.89^{(1)} \quad \mathrm{P}$ & $=0.032$ \\
\hline $\begin{array}{l}\text { Attending last year CME } \\
\text { on cervical cancer or its } \\
\text { screening }\end{array}$ & & & & & & \\
\hline - $\quad$ Yes & $14(38.9 \%)$ & $22(61.1 \%)$ & $78(80.4 \%)$ & $19(19.6 \%)$ & $18(50.0 \%)$ & $18(50.0 \%)$ \\
\hline - $\quad$ No & $62(63.9 \%)$ & $35(36.1 \%)$ & $4(100.0 \%)$ & $0(0.0 \%)$ & $72(74.2 \%)$ & $25(25.8 \%)$ \\
\hline - don't remember & $3(75.0 \%)$ & $1(25.0 \%)$ & $33(91.7 \%)$ & $3(8.3 \%)$ & $4(100.0 \%)$ & $0(0.0 \%)$ \\
\hline & $\chi^{2}=7.22^{(1)}$ & $=0.027$ & $\chi^{2}=3.255 \quad \mathrm{P}$ & $=0.196$ & $\chi^{2}=9.86^{(1)} \mathrm{F}$ & $=0.007$ \\
\hline $\begin{array}{l}\text { (2) Chi square likeliho } \\
\text { have expected coun }\end{array}$ & d ratio was & lculated inst & d of Pearson & s chi square & ce more than & $25 \%$ of cells \\
\hline
\end{tabular}




\section{Discussion:-}

Globally, cervical cancer is the $2^{\text {nd }}$ most common cancer in women. Screening for cancer cervix is an important and efficient way to early detect, prevent and delay the progress of this cancer. Primary health care workers should play important roles as health educators and promoters. Therefore, it is essential that PHC physicians should have satisfactory knowledge and high compliance toward cancer cervix screening recommendations (Can et al., 2014). ${ }^{(31)}$

Results of the present study showed that participants' knowledge grades regarding cervical cancer were quite suboptimal, where $42.3 \%$ were knowledgeable regarding cancer cervix, $16.1 \%$ were knowledgeable regarding screening guidelines, and $31.4 \%$ had overall knowledge. Moreover, areas of participant physicians' poor knowledge were identified. Those regarding cancer cervix were related to smoking and poor hygiene as risk factors for cervical cancer, while those related to cancer cervix screening guidelines were "the only screening method is Pap test" and "a 30 years old female can be screened by Pap test with HPV testing every five years".

These findings, despite unexpected, are in agreement with those of several studies. In Jordan, Obeidat et al. (2012) reported that half of Jordanian female health care workers could not recognize the importance of cervical smear in relation to the prevention of cervical cancer. Areas of poor knowledge were related to that association between HPV infection and cervical cancer. ${ }^{(32)}$

In Mexico, Gonzalez-Losa et al. (2009) reported that family physicians had low knowledge regarding cancer cervix screening. ${ }^{(33)}$

Moreover, Holland-Barkis et al. (2006), in Texas, USA, found wide variations in physicians' knowledge and adherence to cervical cancer screening guidelines, despite their general agreement that knowledge of, and adherence to, these guidelines are important. ${ }^{(34)}$

In Jeddah, Sait (2011) reported that about one-third of non-gynecologist physicians could not identify HPV as a cause of cervical cancer, $33.8 \%$ believed that cervical cancer is curable. ${ }^{(35)}$

Reasons for the unsatisfactory knowledge among PHC physicians in Makkah Al-Mokarramah were explored in the present study. It has been showed that, although most participant physicians $(89.1 \%)$ heard about Pap smear testing, only $68(49.6 \%)$ updated their knowledge by reading about cervical screening guidelines during the last year, while only $26.3 \%$ of participants attended CME on cervical cancer and its screening during the last year. Besides, only 50 PHC physicians $(36.5 \%$ ) had an MOH-protocol for cervical cancer screening. Among those 50 physicians, only 22 $(44 \%)$ had access to that protocol.

These findings indicate the pressing necessity for directing efforts to promote knowledge of PHC physicians in Makkah Al-Mokarramah about early diagnosis of cancer cervix. They should be provided with standard guidelines and to be encouraged toward regularly attending courses/workshops on prevention and management of cancer cervix.

Findings of the present study are in agreement with those of Hyacinth et al. (2012), in North central Nigeria, reported limited knowledge among health workers regarding Pap smear. Only 38\% reported having heard about the Pap smear test, and the main reason for the limited knowledge was they were not well-trained for that purpose. ${ }^{(36)}$

In Jeddah, Saudi Arabia, Sait et al. (2011) concluded that physicians need further education regarding the available screening, prevention for HPV, and cervical cancer. ${ }^{(31)}$

However, In Qatar, majority of the female healthcare workers were aware of cervical cancer screening $(97.8 \%)$ and had fair to good knowledge about cervical cancer screening. ${ }^{(37)}$

The World Health Organization (2006) emphasized PHC professionals' role in cervical cancer screening. It has been observed that in countries with high prevalence incidence of cancer cervix, women never attend cancer screening because of the inadequate experience or knowledge of these preventive services offered by health care providers. ${ }^{(38)}$ Thus, comprehensive training and re-training of PHC workers are highly needed. 
Investigating practices of PHC physicians in Makkah Al-Mokarramah regarding cancer cervix screening showed that $33.6 \%$ used to ask asymptomatic females about screening for cancer cervix, while only $14.6 \%$ always refer female patients to undergo screening for cancer cervix.

These findings are in agreement with those of Obeidat et al. (2012), who demonstrated the inadequacy of health professionals' practices toward secondary prevention of cervical cancer. ${ }^{(32)}$

Sait (2011) reported that non-gynecologist physicians at the Faculty of Medicine, King Abdulaziz University Hospital presented low proportion of adequate practices related to cancer cervix screening. ${ }^{(35)}$

Mutyaba et al. (2006) reported that lack of cancer cervix screening among medical workers at Mulago Hospital in Uganda, was perpetuated by their lack of knowledge, insufficient resources and the absence of clear screening programs, policies, and guidelines. ${ }^{(39)}$

Bose et al. (2016) recommended that adequate and organized screening services should be made available and accessible at all health care facilities. ${ }^{(40)}$ Jhala and Eltoum (2007) stated that the limited practices of physicians regarding screening for cancer cervix are due to low physicians' level of knowledge about cervical screening, education/specialty of a provider, and the health care system which does not facilitate or encourage screening. ${ }^{(41)}$

Sait et al. (2011) explained that the possible reasons why physicians do not carry out screening for cancer cervix or refer patients for screening could be attributed to their busy practices and/or their lack of knowledge. ${ }^{(35)}$

The present study showed significantly better knowledge grades regarding cancer cervix and also regarding overall knowledge about cancer cervix among participants who were non-Saudi, which could be explained that Saudi Arabia is still more conservative regarding discussion of cervical cancer screening issues and screening in general, as well as those who were Board certified.

These findings indicate that better qualified PHC physicians are more knowledgeable regarding screening for cancer cervix. Moreover, although the Saudi Ministry of Health usually hires qualified and experienced non-Saudi PHC physicians, there is an obvious need to provide better education and training for Saudi PHC physicians regarding screening for cancer cervix.

However, family medicine diploma certified physicians had the lowest scores in overall knowledge, which may be attributed to the short duration of their training or a defect in their curriculum

In another study carried out among primary health care physicians in Qatar, educational background of the respondents and center`s characteristics were found to significantly affect the awareness and knowledge of cervical screening. ${ }^{(37)}$

Results of the current study showed that PHC participants' knowledge grades about cancer cervix and overall knowledge were significantly better among physicians who work at JCI/CBAH-accredited PHCCs, perform periodic health examination, which includes Pap-testing, receive higher numbers of patients, or which have higher numbers of physicians.

The importance of institutional accreditation regarding health care related to cancer cervix prevention has been emphasized by the British Columbia Cancer Agency (2017). They stated that the Cancer Cervix Screening Laboratory demonstrates an ongoing commitment to providing quality patient care by following internationally recognized standards of excellence in lab practices ${ }^{(42)}$

These findings reflect that role played by the PHCC structure and the positive impact of better equipped PHCCs on PHC physicians' knowledge regarding cancer cervix. It is clear that PHCCs which are JCI/CBAH-accredited, which included Pap-testing in their periodic health examination, which is capable of receiving more patients and employ more physicians offer better chances to enhance their physicians' knowledge or maybe accredited to the employment of highly qualified physicians in such PHCC.

It has been noted that Pap smear is structurally demanding. In most countries of Sub-Saharan Africa, most health care facilities are not properly equipped to provide Pap Smear at the population level (Mutyaba et al. 2007). ${ }^{(43)}$ 
These findings are in agreement with those reported by several studies. Mutyaba et al. (2007) noted that Papsmear is structurally demanding. ${ }^{(44)}$ In several developing countries, most health care facilities are not properly equipped to provide Pap Smear at population level.

Paz-Soldán et al. (2012) added that the widespread lack of resources at health care facilities is a major structural barrier against screening for and treatment of cervical cancer in Lima, Peru. ${ }^{(45)}$

PHC participants' knowledge grades regarding cancer cervix and overall knowledge were significantly better among physicians who heard about Pap smear and those who attended CME on cervical cancer and its screening. In addition, participants' overall knowledge grades about cancer cervix were significantly better among physicians who ask asymptomatic patients about cancer cervix screening and those who had their information from online sources. Moreover, PHC physicians who had their information from online sources were also significantly more knowledgeable regarding cancer cervix screening guidelines than those who obtained their information from other sources.

These findings, which are in accordance with those reported by several other studies, denote the importance of encouraging PHC physicians to update their knowledge and to promote their practices regrading cancer cervix screening.

In Nigeria, Bose et al. (2016) reported a significant association between PHC physicians' knowledge about cancer cervix and their practices regarding its screening. ${ }^{(40)}$

Roland et al. (2010) highlighted the importance of online sources for raising awareness and knowledge of physician toward cancer cervix screening. They concluded that online CME activities generally support updated guidance for screening for cancer cervix but need more information on counseling patients ${ }^{(46)}$. Nguyen et al. (2000) added that CME not only leads to positive changes in knowledge among physicians, but also changes in their practices. ${ }^{(47)}$

Among strengths of the present study, it included a sample representing primary health care physicians working at Ministry of Health PHCC in the three supervisory sectors inside Makkah AL-Mokarramah city. Also, the use of a self-administered questionnaire instead of interviewing physicians which have a high potential for interviewer bias. However, this study has some limitations. It involved doctors from specific PHCC in Makkah. Also; it does not involve physicians in other primary health care centers, as well as physicians from different regions of Saudi Arabia as physicians from other primary health centers, may have characteristics that distinguished them from our sample in relation to their level of knowledge of cervical cancer and its screening. These data are cross-sectional and limit our ability to make causal inference among studied associated factors.

limitations: -

- Lack of cooperation from some of the physicians.

- Widespread of PHCC in Urban part of Makkah Al-Mokarramh.

\section{Conclusions:-}

Based on the findings of this study, the following can be concluded:

- Knowledge grades of PHC physicians in Makkah Al-Mokarramah regarding cancer cervix and its screening are suboptimal.

- Areas of participant physicians' poor knowledge regarding cancer cervix are related to smoking and poor hygiene as risk factors for cervical cancer, while those related to cancer cervix screening guidelines are "the only screening method is Pap test" and "a 30 years old female can be screened by Pap test with HPV testing every five years".

- Reasons for unsatisfactory knowledge among PHC physicians in Makkah Al-Mokarramah are the low proportion of physicians who update their knowledge through reading and/or attending CME, in addition to the lack of availability of and access to MOH-protocol for cervical cancer screening.

- Practices of PHC physicians in Makkah Al-Mokarramah regarding cancer cervix screening are low. Few physicians ask asymptomatic females about screening for cancer cervix or refer female patients to undergo screening. 
- There are significantly better knowledge grades regarding cancer cervix and also regarding overall knowledge about cancer cervix among participants who are non-Saudi, those who are Board certified or Diploma certified, among physicians who work at JCI/CBAH-accredited PHCCs, perform periodic health examination, which apply Pap-testing, receive higher numbers of patients, or which have higher numbers of physicians, among physicians who heard about Pap smear and those who attended CME on cervical cancer and its screening and those who obtain information from online sources.

\section{Recommendations:-}

Based on the findings of this study, the following can be recommended:

- There is a pressing necessity to promote knowledge of PHC physicians in Makkah Al-Mokarramah about early diagnosis of cancer cervix.

- $\quad$ PHC physicians should be provided with standard guidelines on cancer cervix screening.

- $\quad$ PHC physicians should be encouraged toward regularly attending CME courses/workshops on prevention and management of cancer cervix.

- To provide the necessary subscription for online access of PHC physicians to specialized medical databases as an important source for knowledge and continuing medical education.

- $\quad$ PHC centers should be sufficiently equipped to undergo cancer cervix screening.

- The undergraduate medical curriculum in Saudi Arabia should enforce levels of prevention, especially screening for cancer.

- To encourage newly graduated PHC physicians to apply for Family Medicine Board certification.

- To extend the CEBAH/JCI accreditation to includes more PHC centers.

- Further nationwide studies are needed to be conducted in areas other than Makkah Al-Mokarramah to explore PHC physicians' knowledge and practices regarding screening for cancer cervix and other important diseases, e.g., breast and colon cancers.

\section{References:-}

1. GLOBOCAN. GLOBOCAN Cancer Fact Sheets: Cervical cancer [Internet]. [cited 2016 Jan 8]. Available from: http://globocan.iarc.fr/old/FactSheets/cancers/cervix-new.asp

2. Council HS. Saudi Cancer Registry. 2010 [Internet]. 2010;24-6. Available from: http://www.chs.gov.sa/Ar/mediacenter/NewsLetter/2010 Report (1).pdf

3. Walboomers JM, Jacobs M V, Manos MM, Bosch FX, Kummer JA, Shah K V, et al. Human papillomavirus is a necessary cause of invasive cervical cancer worldwide. J Pathol [Internet]. 1999 Sep [cited 2015 Jan 11];189(1):12-9. Available from: http://www.ncbi.nlm.nih.gov/pubmed/10451482

4. Turki R, Sait K, Anfinan N, Sohrab SS, Abuzenadah AM. Prevalence of human papillomavirus in women from Saudi Arabia. Asian Pac J Cancer Prev [Internet]. 2013 Jan [cited 2016 Jan 7];14(5):3177-81. Available from: http://www.ncbi.nlm.nih.gov/pubmed/23803100

5. AlObaid A, Al-Badawi IA, Al-Kadri H, Gopala K, Kandeil W, Quint W, et al. Human papillomavirus prevalence and type distribution among women attending routine gynecological examinations in Saudi Arabia. BMC Infect Dis [Internet]. 2014 Jan 14 [cited 2015 Dec 13];14(1):643. Available from: http://bmcinfectdis.biomedcentral.com/articles/10.1186/s12879-014-0643-8

6. Alsbeih G, Ahmed R, Al-Harbi N, Venturina LA, Tulbah A, Balaraj K. Prevalence and genotypes' distribution of human papillomavirus in invasive cervical cancer in Saudi Arabia. Gynecol Oncol [Internet]. 2011 Jun 1 [cited 2016 Jan 8];121(3):522-6. Available from: http://www.ncbi.nlm.nih.gov/pubmed/21353296

7. Society of Gynecologic Oncologists Education Resource Panel Writing group C, Collins Y, Einstein MH, Gostout BS, Herzog TJ, Massad LS, et al. Cervical cancer prevention in the era of prophylactic vaccines: a preview for gynecologic oncologists. Gynecol Oncol [Internet]. 2006 Sep [cited 2017 Mar 4];102(3):552-62. Available from: http://www.ncbi.nlm.nih.gov/pubmed/16979432

8. Berrington De González A, Green J. Comparison of risk factors for invasive squamous cell carcinoma and adenocarcinoma of the cervix: Collaborative reanalysis of individual data on 8,097 women with squamous cell carcinoma and 1,374 women with adenocarcinoma from 12 epidemiological studies. Int J Cancer [Internet]. $2007 \mathrm{Feb}$ 15 [cited 2017 Apr 18];120(4):885-91. Available from: http://doi.wiley.com/10.1002/ijc.22357

9. Appleby P, Beral V, Berrington De González A, Colin D, Franceschi S, Goodill A, et al. Carcinoma of the cervix and tobacco smoking: Collaborative reanalysis of individual data on 13,541 women with carcinoma of the cervix and 23,017 women without carcinoma of the cervix from 23 epidemiological studies. Int J Cancer [Internet]. 2006 Mar 15 [cited 2017 Apr 18];118(6):1481-95. Available from: http://doi.wiley.com/10.1002/ijc.21493

10. International Collaboration of Epidemiological Studies of Cervical Cancer B, Appleby P, Beral V, Berrington de 
González A, Colin D, Franceschi S, et al. Cervical cancer and hormonal contraceptives: collaborative reanalysis of individual data for 16,573 women with cervical cancer and 35,509 women without cervical cancer from 24 epidemiological studies. Lancet (London, England) [Internet]. 2007 Nov 10 [cited 2017 Mar 4];370(9599):1609-21. Available from: http://www.ncbi.nlm.nih.gov/pubmed/17993361

11. Li N, Franceschi S, Howell-Jones R, Snijders PJF, Clifford GM. Human papillomavirus type distribution in 30,848 invasive cervical cancers worldwide: Variation by geographical region, histological type and year of publication. Int $\mathrm{J}$ Cancer [Internet]. 2011 Feb 15 [cited 2017 Mar 4];128(4):927-35. Available from: http://doi.wiley.com/10.1002/ijc. 25396

12. Mutter G, Prat J. Pathology of the Female Reproductive Tract [Internet]. Churchill Livingstone/Elsevier; 2014 [cited 2017 Apr 18]. 1066 p. Available from: https://books.google.com.sa/books?id=ab545XLMBEC\&pg=PA219\&dq=Cervical+cancer+usually+originate+at+transformation+zone \&hl=en\&sa=X\&redir_esc $=\mathrm{y} \# \mathrm{v}$ $=$ onepage $\& \mathrm{q}=$ Cervical cancer usually originate at transformation zone $\& \mathrm{f}=\mathrm{false}$

13. DiSaia PJ CW. Clinical Gynecologic Oncology, 7th ed. 7th ed. Philadelphia: Mosby Elsevier; 2007. p. 55.

14. Chang AE, Hayes DF, Pass HI, Stone RM, Ganz PA, Kinsella TJ, et al. Oncology: An evidence-based approach. Oncol An Evidence-Based Approach [Internet]. 2006 [cited 2017 Apr 17];(January):1-2005. Available from: https://books.google.com.sa/books?id=vxh6u1-

ETk0C\&pg=PA875\&dq=physical+examination+in+cervical+cancer\&hl=en\&sa=X\&redir_esc=y\#v=onepage \&q=phy sical examination in cervical cancer\& $\mathrm{f}=\mathrm{false}$

15. Partridge EE, Abu-Rustum NR, Campos SM, Fahey PJ, Farmer M, Garcia RL, et al. Cervical Cancer Screening. J Natl Compr Cancer Netw [Internet]. 2010 Dec [cited 2017 Apr 17];8(12):1358-86. Available from: http://www.ncbi.nlm.nih.gov/pubmed/21147902

16. Pecorelli S. FIGO COMMITTEE ON GYNECOLOGIC ONCOLOGY Revised FIGO staging for carcinoma of the vulva, cervix, and endometrium. Int J Gynecol Obstet [Internet]. [cited 2017 Mar 5];105:103-4. Available from: http://www.csh.org.tw/dr.tcj/Educartion/Cancer center/PDF/2010/FIGO staging revision 2009.pdf

17. TNM A. cervical cancer staging TNM. [cited 2017 Mar 5]; Available from: https://cancerstaging.org/referencestools/quickreferences/Documents/CervixMedium.pdf

18. Greer BE, Koh W-J, Abu-Rustum NR, Apte SM, Campos SM, Chan J, et al. Cervical cancer. J Natl Compr Canc Netw [Internet]. 2010 Dec [cited 2017 Apr 18];8(12):1388-416. Available from: http://www.ncbi.nlm.nih.gov/pubmed/21147903

19. World Health Organisation. Comprehensive Cervical Cancer Control [Internet]. WHO Library Cataloguing-inPublication Data. 2014. Available from: http://apps.who.int/iris/bitstream/10665/144785/1/9789241548953_eng.pdf

20. USPTF. Final Recommendation Statement: Cervical Cancer: Screening - US Preventive Services Task Force

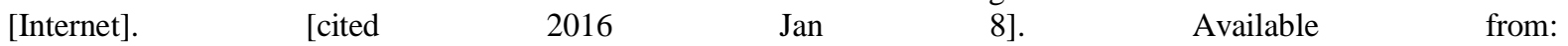
http://www.uspreventiveservicestaskforce.org/Page/Document/RecommendationStatementFinal/cervical-cancerscreening

21. Profile on: George Papanikolaou, creator of the cervical Pap smear test | To Muse and Carouse on WordPress.com [Internet]. [cited 2016 Jan 8]. Available from: http://solangefrancois.com/2012/03/04/profile-on-georgepapanikolaou-creator-of-the-cervical-pap-smear-test/

22. Committee on Practice Bulletins - Gynecology. Practice Bulletin No. 168: Cervical Cancer Screening and Prevention. Obstet Gynecol [Internet]. 2016 Oct [cited 2017 Mar 7];128(4):e111-30. Available from: http://content.wkhealth.com/linkback/openurl?sid=WKPTLP:landingpage\&an=00006250-201610000-00058

23. Castle PE, Katki HA. Benefits and risks of HPV testing in cervical cancer screening. Lancet Oncol [Internet]. 2010 Mar [cited 2016 Jan 8];11(3):214-5. Available from: http://www.ncbi.nlm.nih.gov/pubmed/20089448

24. Mayrand M-H, Duarte-Franco E, Rodrigues I, Walter SD, Hanley J, Ferenczy A, et al. Human Papillomavirus DNA versus Papanicolaou Screening Tests for Cervical Cancer. N Engl J Med [Internet]. 2007 Oct 18 [cited 2017 Apr 17];357(16):1579-88. Available from: http://www.nejm.org/doi/abs/10.1056/NEJMoa071430

25. Sasieni P, Castanon A, Cuzick J. Effectiveness of cervical screening with age: population based case-control study of prospectively recorded data. BMJ [Internet]. 2009 Jan [cited 2016 Jan 8];339:b2968. Available from: http://www.pubmedcentral.nih.gov/articlerender.fcgi?artid=2718082\&tool=pmcentrez\&rendertype=abstract

26. Quinn M, Babb P, Jones J, Allen E. Effect of screening on incidence of and mortality from cancer of cervix in England: evaluation based on routinely collected statistics. BMJ [Internet]. 1999 Apr 3 [cited 2016 Jan 8];318(7188):904-8. Available from: http://www.pubmedcentral.nih.gov/articlerender.fcgi?artid=27810\&tool=pmcentrez\&rendertype=abstract

27. CDC. Cervical Cancer Screening Guidelines for Average-Risk Women. [cited 2017 Jun 20]; Available from: https://www.cdc.gov/cancer/cervical/pdf/guidelines.pdf

28. Saslow D, Castle PE, Cox JT, Davey DD, Einstein MH, Ferris DG, et al. American Cancer Society Guideline for human papillomavirus (HPV) vaccine use to prevent cervical cancer and its precursors. CA Cancer J Clin [Internet]. 2007 Jan 1 [cited 2017 Mar 8];57(1):7-28. Available from: http://doi.wiley.com/10.3322/canjclin.57.1.7 
29. Saslow D, Solomon D, Lawson HW, Killackey M, Kulasingam SL, Cain J, et al. American Cancer Society, American Society for Colposcopy and Cervical Pathology, and American Society for Clinical Pathology screening guidelines for the prevention and early detection of cervical cancer. CA Cancer J Clin [Internet]. 2012 [cited 2017 Mar 8];62(3):147-72. Available from: http://www.ncbi.nlm.nih.gov/pubmed/22422631

30. Quadrivalent vaccine against human papillomavirus to prevent high-grade cervical lesions. N Engl J Med [Internet]. 2007 May 10 [cited 2016 Jan 9];356(19):1915-27. Available from: http://www.ncbi.nlm.nih.gov/pubmed/17494925

31. Huseyin Can1,2*, Ozgur Erdem3, Coskun Oztekin4, Sercan Bulut Celik4, Mete Onde5, Tahsin Celepkolu6, Kurtulus Ongel1 7. Are Primary Health Care Workers Aware of Cervical Cancer Risk? Asian Pacific J Cancer Prev Asian Pac J Cancer Prev. 2014;15(1516):6669-71.

32. OBEIDAT BR, AMARIN ZO, ALZAGHAL L. Awareness, practice and attitude to cervical Papanicolaou smear among female health care workers in Jordan. Eur J Cancer Care (Engl) [Internet]. 2012 May [cited 2017 Jan 7];21(3):372-6. Available from: http://www.ncbi.nlm.nih.gov/pubmed/22050559

33. del Refugio Gonzalez-Losa M, Gongora-Marfil GK, Puerto-Solis M. Knowledge about cervical cancer screening among family physicians: cross-sectional survey. J Eval Clin Pract [Internet]. 2009 Apr [cited 2017 Jan 13];15(2):289-91. Available from: http://doi.wiley.com/10.1111/j.1365-2753.2008.00996.x

34. Holland-Barkis P, Forjuoh SN, Couchman GR, Capen C, Rascoe TG, Reis MD. Primary care physicians' awareness and adherence to cervical cancer screening guidelines in Texas. Prev Med (Baltim). 2006;42(2):140-5.

35. Sait KH. Knowledge, attitudes, and practices regarding cervical cancer screening among physicians in the Western Region of Saudi Arabia. [Internet]. Vol. 32, Saudi Medical Journal. 2011 [cited 2016 Jan 8]. p. 1155-60. Available from: http://www.smj.org.sa/index.php/smj/article/view/7307

36. Hyacinth HI, Adekeye OA, Ibeh JN, Osoba T. Cervical Cancer and Pap Smear Awareness and Utilization of Pap Smear Test among Federal Civil Servants in North Central Nigeria. PLoS One. 2012;7(10):1-8.

37. Dr. Amal Alali (1), Dr. Mohamed Salem. Knowledge, attitudes and practices regarding cervical cancer screening among Female health care workers in primary healthcare in Qatar. MIDDLE EAST J Fam Med [Internet]. [cited 2016 Dec 30];VOLUME 14(8). Available from: http://www.mejfm.com/Ocober 2016/Cervical cancer.pdf

38. World Health Organization. Comprehensive Cervical Cancer Control. A guide to essential practice. WHO Libr Cat Data [Internet]. $2006 \quad$ [cited 2017 Apr 18];1-284. Available from: https://books.google.com.sa/books?id=a4cYeKpbXH8C\&printsec=frontcover\&dq=treatment+of+cervical+cancer\&hl $=$ en $\& \mathrm{sa}=\mathrm{X} \&$ redir_esc $=\mathrm{y} \# \mathrm{v}=$ onepage $\& \mathrm{q}=$ treatment of cervical cancer $\& \mathrm{f}=$ false

39. Mutyaba T, Mmiro FA, Weiderpass E. Knowledge, attitudes and practices on cervical cancer screening among the medical workers of Mulago Hospital, Uganda. BMC Med Educ [Internet]. 2006 Mar 1 [cited 2017 Jun 17];6(1):13. Available from: http://www.ncbi.nlm.nih.gov/pubmed/16509979

40. Bose M, Titilope A, Andrew S, Titilayo O, Ololade O, Jonathan D. - Cancer of the cervix and Cervical screening: current knowledge, attitude and practice of primary health care workers in Ikenne Local Government Area Ogun State, Nigeria. \&quot; IOSR J Nurs Heal Sci [Internet]. [cited 2017 Jan 6];5(5):2320-1940. Available from: www.iosrjournals.org

41. Jhala D, Eltoum I. Barriers to adoption of recent technology in cervical screening. Cytojournal [Internet]. 2007 Aug 16 [cited 2017 Jun 17];4(1):16. Available from: http://www.ncbi.nlm.nih.gov/pubmed/17705820

42. Riddell L, Swalwell-franks A. Screening for Cancer of the Cervix An Office Manual for Health Professionals. 2010; Available from: www.screeningbc.ca/cervix

43. Lim JNW, Ojo AA. Barriers to utilisation of cervical cancer screening in Sub Sahara Africa: a systematic review. Eur J Cancer Care (Engl) [Internet]. 2017 Jan 1 [cited 2017 Jun 19];26(1):e12444. Available from: http://doi.wiley.com/10.1111/ecc. 12444

44. Mutyaba T, Faxelid E, Mirembe F, Weiderpass E. Influences on uptake of reproductive health services in Nsangi community of Uganda and their implications for cervical cancer screening. Reprod Health [Internet]. 2007 [cited 2017 Jun 17];4(1):4. Available from: http://www.reproductive-health-journal.com/content/4/1/4

45. Paz-Soldán VA, Bayer AM, Nussbaum L, Cabrera L. Structural barriers to screening for and treatment of cervical cancer in Peru. Reprod Health Matters [Internet]. 2012 Dec [cited 2017 Jun 17];20(40):49-58. Available from: http://www.ncbi.nlm.nih.gov/pubmed/23245408

46. Roland KB, Larkins TL, Benard VB, Berkowitz Z, Saraiya M. Content analysis of continuing medical education for cervical cancer screening. J Womens Health (Larchmt) [Internet]. 2010 Apr [cited 2017 Jun 17];19(4):651-7. Available from: http://www.ncbi.nlm.nih.gov/pubmed/20350199

47. Lai KQ, Nguyen TT, Mock J, McPhee SJ, Doan HT, Pham TH. Increasing Vietnamese-American physicians' knowledge of cervical cancer and pap testing: Impact of continuing medical education programs. Ethn Dis. 2004;14(3 SUPPL. 1). 